\title{
Recent trends in the development of hydrogel therapeutics for the treatment of central nervous system disorders
}

\author{
Qing Li $i^{1,2}$, Xinxin Shao ${ }^{1,2}$, Xianglin Dai ${ }^{2}$, Qiong Guo ${ }^{1,2}$, Bolei Yuan ${ }^{1,2}$, Ying Liu $\mathbb{C}^{2}$ and Wei Jiang $\mathbb{C}^{1,2}$
}

\begin{abstract}
The central nervous system (CNS) controls the acquisition and processing of peripheral information to manage the behaviors of organisms. CNS disorders, including CNS injuries, neurodegenerative diseases, and brain tumors, are devastating and can cause life-long disabilities. Despite the advanced medical interventions in the modern era of biomedical technology, noninvasive therapeutic strategies are still limited for the prevention or reversal of disease progression. Such scarcity is mainly caused by intricate pathological mechanisms and the unique biological microenvi ronment of the CNS. Thus, the development of a carrier that promotes the delivery of therapeutic agents into the brain is vital. Hydrogels, as a synthetic or natural platform with a porous three-dimensional structure, can be applied as desirable drug delivery vehicles and cell transportation platforms. This review focuses on the most recent advance ments in hydrogel-based therapies for the treatment of CNS disorders, including brain injury, spinal cord injury, neurodegenerative diseases, and brain tumors.
\end{abstract}

\section{Introduction}

The central nervous system (CNS) plays an irreplaceable role in health and well-being ${ }^{1}$. The brain is one of the most vital organs and a delicate organ of the CNS; it interrelates the input from the external environment and processes the information to enable responses ${ }^{2}$. The spinal cord is another significant component of the CNS that performs multiple important functions, such as motion, information conduction and reflexes ${ }^{3}$. CNS disorders account for up to $6.3 \%$ of all diseases worldwide owing to the increasing aging of the population, which causes major health issues and increases costs for medical and nursing care ${ }^{3}$. Apart from aging, several pathological states, such as neurodegenerative diseases (NDs), neurotraumas, and brain tumors, can affect the CNS, leading to gradual cell

Correspondence: Wei Jiang (weijiang@zzu.edu.cn)

${ }^{1}$ Department of Molecular Pathology, Application Center for Precision Medicine, The Second Affiliated Hospital of Zhengzhou University, Zhengzhou, Henan 450052, China

${ }^{2}$ Center for Precision Medicine, Academy of Medical Sciences, Zhengzhou University, Zhengzhou 450001, China degeneration, neuronal death, severe disabilities, and ultimately death ${ }^{4}$. Unfortunately, the limited regeneration capability of CNS neurons can lead to permanent functional loss, posing a major challenge in the treatment of CNS injuries or disorders ${ }^{5,6}$. Regenerative medicine, which has always been a popular topic in neurology and a key treatment method for CNS injuries, aims to substitute or regenerate cells, tissues, or even organs to restore functions ${ }^{7}$. Two main strategies can be implemented in regenerative medicine: (1) the delivery of stem cells into the CNS and the realization of their differentiation and integration with the host tissue ${ }^{8}$ and (2) the delivery of therapeutic drugs to stimulate the regeneration of endogenous cells ${ }^{5,9-11}$. However, the unique anatomical and physiological structure of the CNS could compromise the therapeutic effect of current therapies ${ }^{12}$. For example, difficulties are encountered in the traditional oral or intravenous administration of therapeutic agents to the CNS because of limited penetration through the blood-brain barrier (BBB) and the blood-cerebrospinal fluid barrier $(\mathrm{BCSFB})^{13,14}$. The $\mathrm{BBB}$ impedes the entry of

\section{(c) The Author(s) 2022}

(c) (i) Open Access This article is licensed under a Creative Commons Attribution 4.0 International License, which permits use, sharing, adaptation, distribution and reproduction c. in any medium or format, as long as you give appropriate credit to the original author(s) and the source, provide a link to the Creative Commons license, and indicate if changes were made. The images or other third party material in this article are included in the article's Creative Commons license, unless indicated otherwise in a credit line to the material. If material is not included in the article's Creative Commons license and your intended use is not permitted by statutory regulation or exceeds the permitted use, you will need to obtain permission directly from the copyright holder. To view a copy of this license, visit http://creativecommons.org/licenses/by/4.0/. 
potentially harmful substances from the blood to the brain and regulates the exchange of nutrients between the blood and the CNS to maintain the nutritive and immune environment of the $\mathrm{CNS}^{3,15}$. However, this delicate mechanism that controls the homeostatic state of the brain also restricts the passage of various therapeutic molecules ${ }^{16}$. Before entering the CNS, molecules must pass through the $\mathrm{BCSFB}$, which controls the transfer of molecules from the blood to the $\mathrm{CSF}^{17}$. The BCSFB is located at the choroid plexuses, and numerous organicacid-based therapeutics, such as antiviral and antitumor agents, are rejected outside the CSF before they diffuse into the brain parenchyma ${ }^{18}$. Therefore, after systemic administration of therapeutic cells or molecules, only modest concentrations of the therapeutic agents reach the CNS. Thus, high doses or frequent administration of therapeutic agents is necessary to achieve the desired therapeutic effect, but this may aggravate systemic toxicity $^{19}$. To address this contradiction, the most frequently used approach is invasive drug delivery, which includes the disruption of the BBB and surgical methods ${ }^{20}$. The disruption of the $\mathrm{BBB}$ was proposed in the $1960 \mathrm{~s}^{21}$ and can be realized through hypertonic solutions such as mannitol, pharmacological agents ${ }^{22}$ or focused ultrasound ${ }^{17}$. Invasive methods can maximize the drug concentration at the targeted site while minimizing the drug exposure of surrounding tissues. For instance, due to the toxicity of chemotherapeutic drugs for brain tumors, it is necessary to use invasive methods to prevent drugs from entering the systemic circulation ${ }^{23,24}$. In addition, by reducing the injection area to a few millimeters, therapeutic agents can be injected directly into the CNS in a diffusion-dependent manner ${ }^{25}$. Unfortunately, these invasive strategies can induce further neuronal damage and inflammatory reactions. For example, pharmacological drugs such as histamine for increasing the permeability of the $\mathrm{BBB}$ are prone to induce inflammatory effects ${ }^{26}$. Therefore, novel delivery methods and treatment strategies are needed for the treatment of CNS disorders.

At present, owing to their excellent performance, injectable hydrogels have attracted the attention of researchers. Hydrogels are polymeric materials crosslinked by physical or chemical methods. They have high biocompatible properties and water content and thus are suitable for tissue regeneration applications ${ }^{27}$. Moreover, because of their porous characteristics, hydrogels are ideal materials for drug loading. By tuning the crosslink density of hydrogels, the diffusion rate of drugs through the hydrogel matrix can be controlled $^{28}$. The design process of hydrogels should consider some properties, such as thermal sensitivity, biodegradability, and injectability, because these properties are vital for drug-hydrogel and hydrogel-tissue interactions. Fortunately, these properties can be manipulated to better meet the actual requirements of clinical applications.

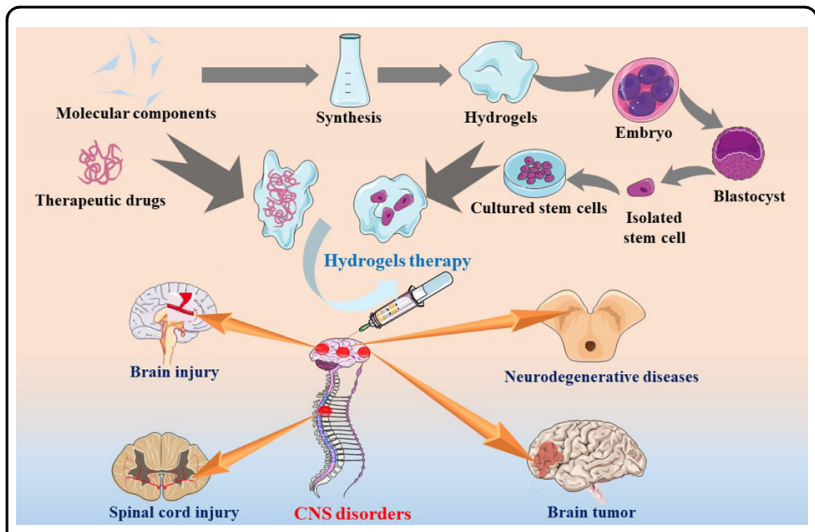

Fig. 1 Hydrogel-based therapies with therapeutic drugs or stem cells for CNS disorders. This review focuses on the most recent advancements of hydrogel-based therapies with therapeutic drugs or stem cells on their applications to the treatment of CNS disorders, including brain injury, spinal cord injury, neurodegenerative diseases, and brain tumors.

Moreover, when integrating hydrogels with other drug delivery systems, such as liposomes and microspheres, carriers with better performance can be created by synergism $^{29}$. Hydrogels enhance particles to reach the injury site and prevent burst release, which is commonly seen with delivery systems, while liposomes and microspheres can extend the release times of hydrophilic agents ${ }^{30}$.

This review aims to summarize the most recent advances in the design and synthesis of hydrogel systems and their application in CNS disorders, including brain injury, spinal cord injury (SCI), NDs, and brain tumors. This review presents the benefits of novel drugs developed based on hydrogels for the treatment of CNS disorders (Fig. 1).

\section{Hydrogels with various components for the treatment of brain injury and nerve damage}

Traumatic brain injury (TBI) and SCI usually occur after incidents such as combat, sports, or strokes, resulting in permanent or chronic damage to the brain and spinal cord. Hydrogels have various advantages when used in the treatment of TBI and SCI, such as sufficient filling of irregular damage sites and the provision of a threedimensional (3D) cell growth environment ${ }^{31}$. Hydrogels with various components, including collagens, hyaluronic acid (HA), chitosan, self-assembled peptides, and decellularized extracellular matrices (ECMs), have been applied in nerve cell regeneration ${ }^{32}$. For a better therapeutic effect, these hydrogels are generally incorporated with drugs, bioactive agents, or even cells ${ }^{33}$.

\section{TBI}

TBI is one of the main causes of disability and death at all ages, and it has long been the focus of brain injury 


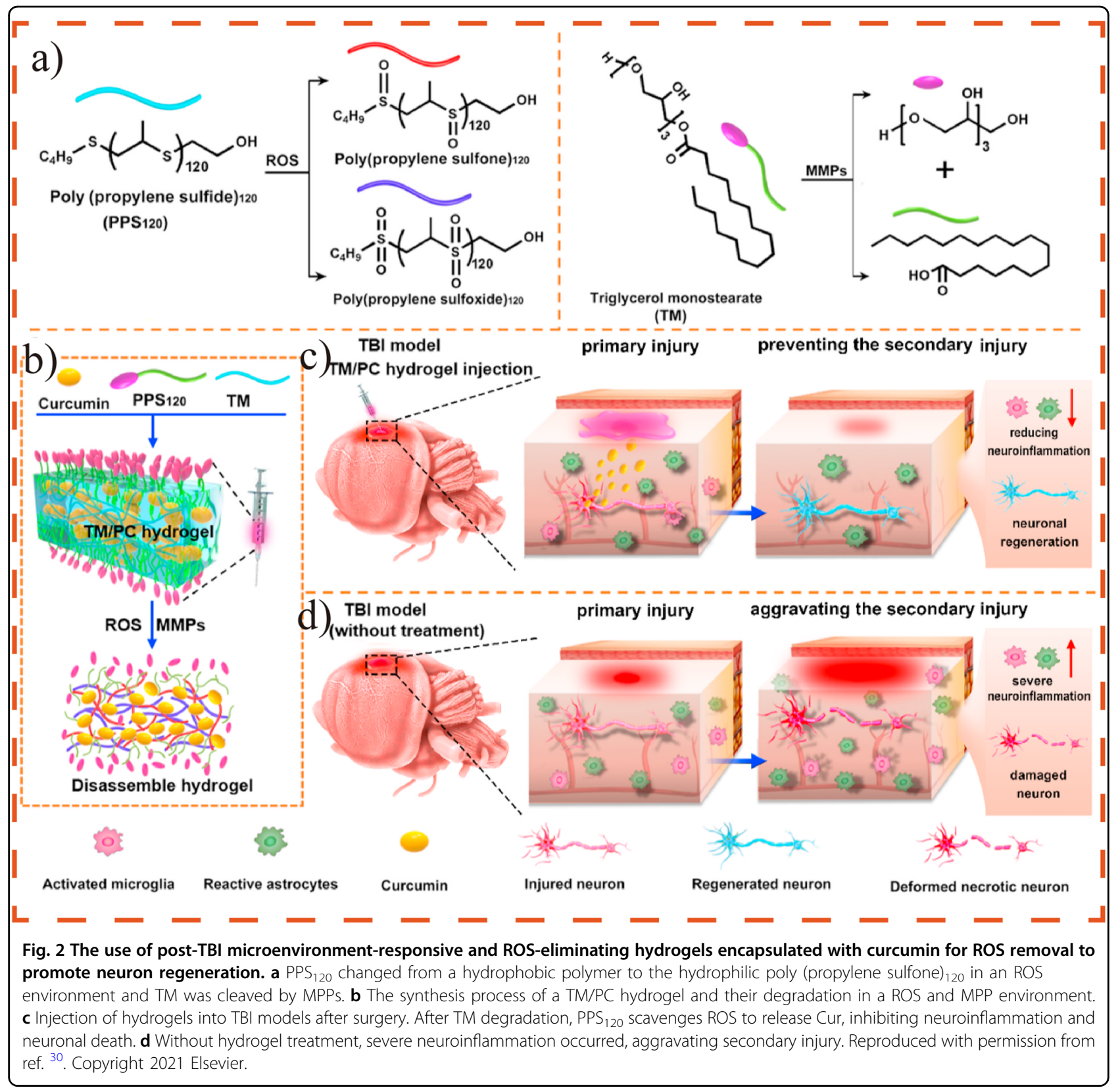

research. After the initial trauma, secondary injury occurs, which can elicit a severe inflammatory response and the breakdown of the BBB, leading to continuous neuronal damage $^{34}$. Recent studies have suggested that TBI-related brain injury is mainly caused by the reactive oxygen species (ROS) induced by secondary damage ${ }^{35}$. Off-theshelf therapeutics, such as the administration of ROS scavengers or growth factors, are restricted because of their poor ability to diffuse across the BBB. Qian et al. reported the use of post-TBI microenvironment-responsive and ROS-eliminating hydrogels encapsulated with curcumin for ROS removal to promote neuron regeneration $^{36}$. In the nanosystem, triglycerol monostearate
(TM) was used to embed hydrophobic molecules, and hydrophobic poly(propylenesulfide) ${ }_{120}\left(\mathrm{PPS}_{120}\right)$ was used as an $\mathrm{H}_{2} \mathrm{O}_{2}$-sensitive and ROS-removing material (Fig. 2a, b) ${ }^{36}$. Synthesized TM/PC hydrogels that contained TM, PPS 120 , and curcumin could be injected into the wound cavity to treat TBI. In TBI models, TM/PC hydrogels efficiently decreased ROS levels and enhanced nervous system functions, showing a desirable protective effect against brain injury (Fig. 2c, d).

In addition to using hydrogels for drug delivery, stem cell replacement treatment (CRT) also has great potential in TBI therapy when designing a suitable microenvironment such as injectable hydrogels for transplanted stem 
cells $^{37}$. Zhang prepared an HA and sodium alginate (SA) scaffold by a tissue engineering method ${ }^{38}$. The high water content made HA/SA hydrogels suitable structures for loading human umbilical cord mesenchymal stem cells (hUC-MSCs). In vivo tests suggested that HA/SA hydrogels are a perfect scaffold for both hUC-MSC survival and the regeneration of endogenous nerve cells, promoting the recovery of nerve functions in patients with TBI. Similarly, Sultan synthesized silk fibroin-based hydrogels for the encapsulation of human mesenchymal stem cells (hMSCs) to treat brain injury ${ }^{39}$. When TBI rat models were treated with the composite via a transseptal approach, encapsulated hMSCs could produce brainderived neurotrophic factor (BDNF) (BDNF-hMSCs), enhancing neuronic functional recovery by reducing the neuronal death rate in the hippocampus. Therefore, stem cell transplantation by hydrogels could be a potential strategy for the clinical treatment of brain disorders.

Compared with direct cell transplantation, ECMs from decellularized tissues may have high treatment potential because of their homologous composition and structure to native tissues and easier transplantation process ${ }^{40}$. More importantly, ECMs from decellularized tissues can be fabricated as injectable gels. A study suggested that hydrogels constructed from brain-tissue-based ECMs could stimulate neurite outgrowth, promoting the tissuespecific functions of ECM-based hydrogels ${ }^{33}$. However, the functions of hydrogels based on different ECMs from nerve tissues are still unknown. Therefore, Zou believed that bioactive factors in nerve tissue ECMs could promote functional recovery after peripheral nerve injury. They constructed hydrogels based on ECMs of decellularized porcine peripheral nerves (DPPNs) and evaluated their efficacy in nerve regeneration compared with Matrigel and collagen I hydrogel ${ }^{33}$. The study illustrated that DPPN-derived hydrogels were more effective in promoting nerve regeneration than Matrigel and collagen I hydrogel, which was consistent with the physiological function of peripheral nerves and the bioinformatics analysis of the system. Based on the above findings, they established a connection between the functions of ECMbased hydrogels and the origins of ECMs. Therefore, they believed that hydrogels containing ECMs of the CNS could be a promising matrix material for the repair of CNS disorders.

Injectable hydrogels based on self-assembled peptides are becoming interesting materials in regenerative medicine because they possess some unique performance advantages. For example, peptide hydrogels can avoid undesirable outcomes by changing the peptide sequence or secondary structure, which cannot be realized by hydrogels based on collagen, chitosan, or $\mathrm{HA}^{38}$. In addition, these biopolymer-based hydrogel scaffolds usually have a high molecular weight, which makes them difficult to characterize by physical methods. Nevertheless, peptide-based hydrogels show varying performances because of their facile synthetic route, flexible modification, and engineering. Therefore, several studies have suggested the high potential of hydrogels crafted from short peptides and peptide derivatives. For example, Wang et al. developed peptide hydrogels self-assembled by the peptide sequences RADA16 and SVVTGLR, which could form nanofibers and gel-like scaffolds (Fig. 3a) ${ }^{41}$. Owing to the angiogenic performance of the SVVYGLR motif, peptide-based hydrogels could promote the formation of the interconnected network of endothelial cells,

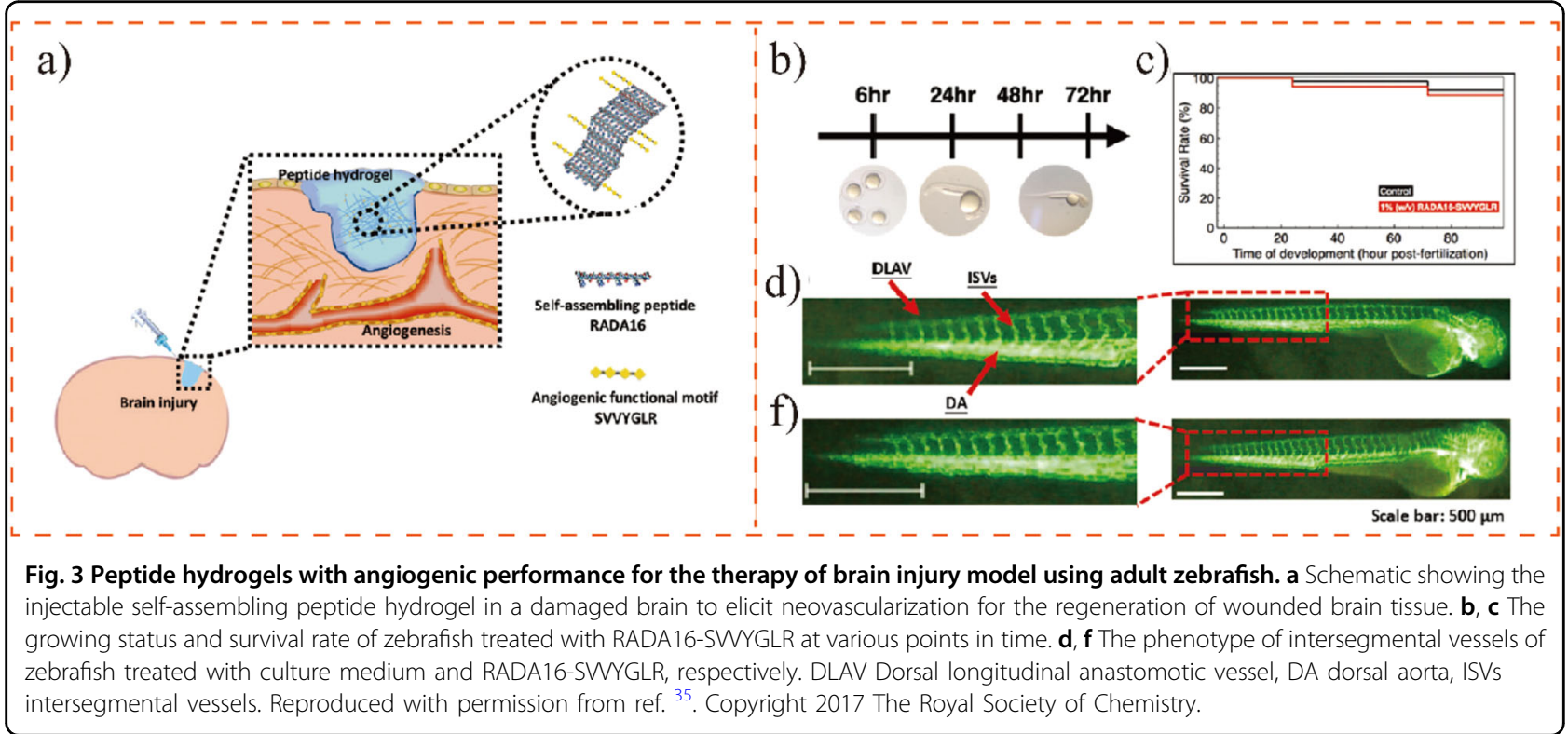



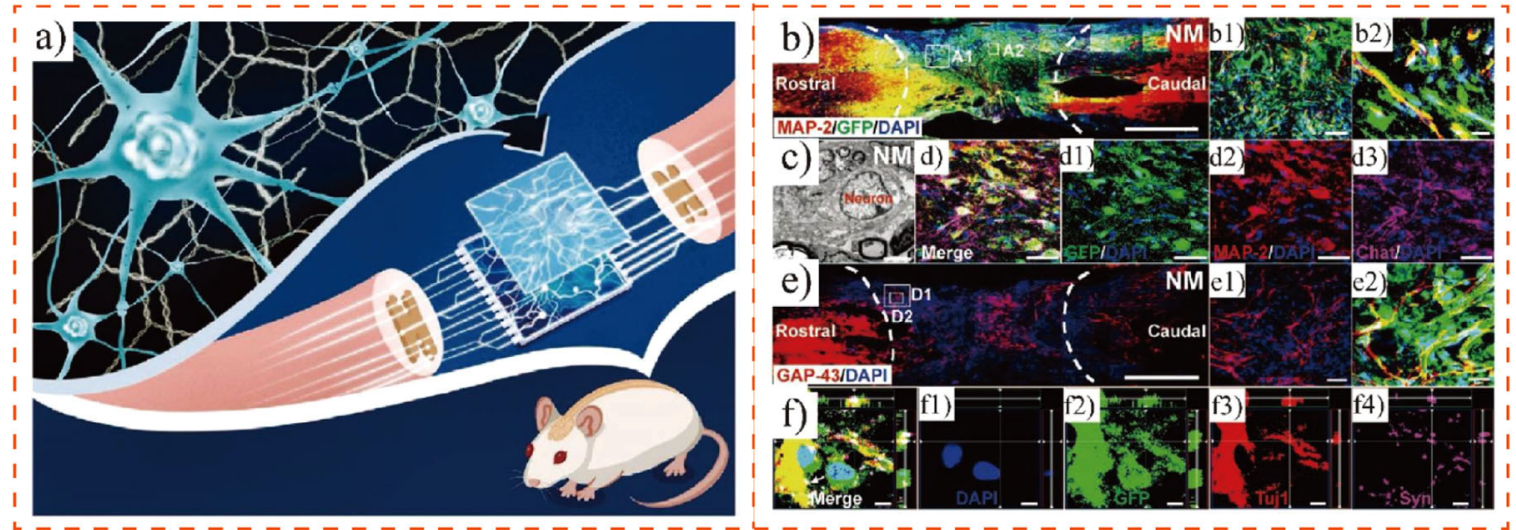

Fig. 4 A kind of DNA supramolecular hydrogels carrying homologous NSCs for functional recovery in SCI mouse models. a Schematic illustration of a newly generated neural network benefited by DNA-based hydrogel at the injury site. b Sagittal section of the spinal cord (coimmunolabeling of GFP, MAP-2, and DAPI) postgrafting. b1, b2 are enlarged pictures from (b). c TEM of a neuron at the injury site postgrafting. d Regenerated motor neuron at the injury site (immunolabeling of GFP, MAP-2, Chat, and DAPI) postgrafting. Scale bars: $20 \mu \mathrm{m}$. e Sagittal section of the spinal cord with GAP-43 and DAPI immunolabeling, Scale bars:1000 $\mu \mathrm{m}$. e1, e2 are the enlarged images from (e) Scale bar: (e1) $50 \mu \mathrm{m}$, (e2) $20 \mu \mathrm{m}$. f Synapse formation at the damaged site with immunolabeling by GFP, Tuj1, SYN, and DAPI. Scale bars: $5 \mu$ m. Reproduced with permission from ref. ${ }^{44}$. Copyright 2021 John Wiley and Sons.

thus enhancing the migration, proliferation, and differentiation of endogenous neural stem cells (Fig. 3d, e). In vivo experiments suggested an ideal therapeutic effect on a brain injury model using adult zebrafish (Fig. 3b, c).

\section{$\mathrm{SCl}$}

SCI, a devastating neurological condition, is closely related to various events, such as ROS formation, vascular changes, and the inflammatory response ${ }^{42,43}$. SCI is associated with complications, such as edema, constipation, and dysuria ${ }^{44}$. Because hydrogels have a structure similar to that of the ECM and can promote axonal growth, the application of hydrogels has enabled regeneration after injury. In addition, hydrogels can load and deliver therapeutic agents, including smallmolecule drugs, chemical drugs, and stem cells, into the injury site ${ }^{45}$.

Combination therapies may be more efficient than single therapies owing to the complex pathology of SCI. Given the neuroprotective effect of minocycline hydrochloride $(\mathrm{MH})$ and the neuroregeneration-promoting effect of paclitaxel (PTX), Nazemi et al. developed alginate hydrogels for the codelivery of MH and PTX to promote tissue regeneration in an SCI rat model ${ }^{46}$. Neural stem cells (NSCs), multipotent cells that play major roles in the production of neural lineage cells, can produce neuronal progeny for SCI therapy ${ }^{47}$. Therefore, the activation of endogenous NSCs for nerve repair and neuroregeneration is anticipated. Yang et al. prudently screened four small molecules, namely, LDN193189 (an inhibitor of activin receptor-like kinase 2/3 (ALK 2/3)), SB431542 (an inhibitor of ALK 5 and TGF- $\beta$ type I receptor), CHIR99021 (an inhibitor of glycogen synthase kinase 3), and P7C3A20 (a highly active analog of P7C3 that has a neuroprotective effect and may promote endogenous repair after TBI), which promote neuronal proliferation and differentiation of spinal cord NSCs ${ }^{48}$. After loading these small molecules into a collagen hydrogel, they can enhance neurogenesis and promote the recovery of locomotion in SCI mouse and rat models.

Similar to TBI, stem cell transplantation to the lesion site is a desirable strategy for SCI treatment. In the past two decades, NSC transplantation technology has been used to treat $\mathrm{SCI}^{49}$. However, this method lacks mechanical support, resulting in the formation of large cavities due to mismatches in mechanical strength. Moreover, the permeability of these transplanting materials has rarely been approved. Considering these issues, Yuan used DNA supramolecular hydrogels with high permeability $^{50}$. DNA hydrogels were used to carry homologous NSCs, repairing a 2-mm-long spinal cord gap in rats (Fig. 4a). The presence of newly generated neurons (Fig. 4b-d) and synapses (Fig. 4e, f) at the injury site indicated the proliferation and differentiation of stem cells after hydrogel administration, reflecting functional recovery in SCI mouse models.

\section{Other types of nerve damage}

Concerning CNS-targeted drug or stem cell delivery, intranasal (IN) administration is a more ideal route than conventional injection methods or the oral route because IN administration can bypass the BBB and improve drug bioavailability $^{51}$. Among various IN delivery formulation components, such as lipid emulsions ${ }^{52}$, surfactants $^{53}$, and 
polymers $^{54}$, thermoresponsive hydrogels are promising because of their temperature-responsive performance to achieve slow release in the body.

Posttraumatic disorder (PTSD), as a psychiatric illness that can affect brain function, is often experienced by patients after abnormal catastrophic events, such as war and earthquakes ${ }^{55}$. Selective serotonin reuptake inhibitors, which are used to treat PTSD clinically, usually have severe side effects. Pang utilized poloxamer as the temperature-sensitive compound and cannabidiol inclusion complex (CBD) as the therapeutic drug to construct temperature-sensitive hydrogels for PTSD therapy ${ }^{56}$. The Hydrogels could extend the retention time of CBD in the nasal cavity, enhancing the brain distribution of CBD and gaining better anti-PTSD effects. Similarly, Wang et al. loaded berberine/hydroxypropyl- $\beta$-cyclodextrin (HP$\beta-C D)$ into a poloxamer-based thermoresponsive hydrogel $^{57}$. After IN delivery of this system, a high concentration of berberine in the brain and an enhanced bioavailability were noted compared with oral administration of berberine/HP- $\beta$-CD. Therefore, IN delivery of the thermoresponsive hydrogel system could elicit a comparative antidepressant effect even at a lower dosage than oral administration of berberine/HP- $\beta-C D$.

\section{Hydrogels as carriers for ND treatment}

NDs, including Parkinson's disease (PD) and Alzheimer's disease (AD), seriously affect the health and quality of life of middle-aged and elderly people ${ }^{58}$. Despite remarkable progress in the localization of the pathology of NDs, the release of therapeutic agents to the pathological site remains difficult in ND treatment ${ }^{59}$. Although stem cell transplantation is a therapeutic strategy, the survival rate after transplantation is unsatisfactory ${ }^{60}$. Therefore, suitable delivery systems are urgently needed. Implanting therapeutic drugs or functional cells in hydrogels for effective delivery to organisms is a modern therapeutic option for the treatment of NDs.

\section{Hydrogel-based drug delivery}

Hydrogels based on peptide self-assembly are promising biomaterials for biomedicine, particularly in treating cerebral disease, because they can form 3D scaffolds for the survival and growth of neurons ${ }^{61}$. An interesting study demonstrated that adjusting the amino acid (AA) sequences and introducing more positively charged AAs lead to the formation of dense fibrillary hydrogel networks, which could simulate the ECM to provide a desirable scaffold for nerve cell regeneration, blood vessel formation, and brain injury recovery ${ }^{61,62}$. Adak developed hydrogels containing microtubule (MT)-stabilizing and neuroprotective residues ${ }^{63}$. Hydrophobic palmitic acid (PA) and hydrophilic residues were also involved in the system. A well-defined hydrogel could be obtained given the amphiphilic characteristics, resulting in crosslinked networks entrapping water molecules. Importantly, a study suggested that the hydrogel could encapsulate anti$\mathrm{AD}$ and anti-PD drugs for wider applications in addition to neuroprotective peptides. Introducing bioactive agents or therapeutic drugs into hydrogels increases the effectiveness in simulating the ECM, contributing to better cell adhesion and proliferation. Activins, as the main transforming growth factors, influence neuroprotective effects. $\mathrm{Li}$ et al. used poly(N-isopropyl acrylamide), a typical thermosensitive polymer ${ }^{64}$, to construct injectable thermosensitive hydrogels to slowly release activin $\mathrm{B}$ and stereotactically injected the system into the striatum of PD mice ${ }^{65}$. As a result of the desired drug release kinetics of activin $\mathrm{B}$, the hydrogel system greatly contributed to substantial cellular protection and symptomatic remission in PD mice.

Clinically, L-dopa is the most effective drug for PD therapy. However, L-dopa has a short half-life and poor bioavailability in vivo, and high-dose and long-term administration will lead to biological resistance, motor complications, movement disorders, and other severe side effects ${ }^{66}$. To obtain the best outcomes of L-dopa therapy, Wang et al. used self-assembling hydrogels to deliver L-dopa for the treatment of $\mathrm{PD}^{66}$. Hydrogels with soft rheological performance and self-healing properties are synthesized by mixing a glutamine amide derivative and benzaldehyde in water, which is compatible with active drugs such as L-dopa. As a result of the appropriate rheological characteristics of the Ldopa-encapsulated hydrogel, IN administration is quite appropriate and has several advantages, such as rapid $\mathrm{L}$-dopa release, prolonged residence times in the nose, and enhanced brain uptake (Fig. 5a). These results were confirmed by animal studies that suggested a better brain distribution and therapeutic efficacy after L-dopaloaded hydrogel administration than after simple L-dopa treatment (Fig. 5b, c).

\section{Functional cell delivery based on hydrogels}

For decades, dopaminergic cell grafts from fetal donors have shown improved neurological recovery in PD models because of their ability to integrate and function after transplanting them into the brain ${ }^{67}$. Nevertheless, the modest survival and regeneration ability after cell transplantation halts clinical progression. As studies have demonstrated that biomaterials can improve the efficiency of tissue regeneration after biomaterial-based cell delivery in PD, Moriarty et al. loaded dopaminergic grafts of younger fetal donors in GDNF-encapsulated hydrogels for their survival, reinnervation, and functional efficacy in PD rats, illustrating the potential of hydrogel biomaterial in neuron protection and repair ${ }^{68}$. Undeniably, the limited graft survival that severely hampers CRTs is mainly 


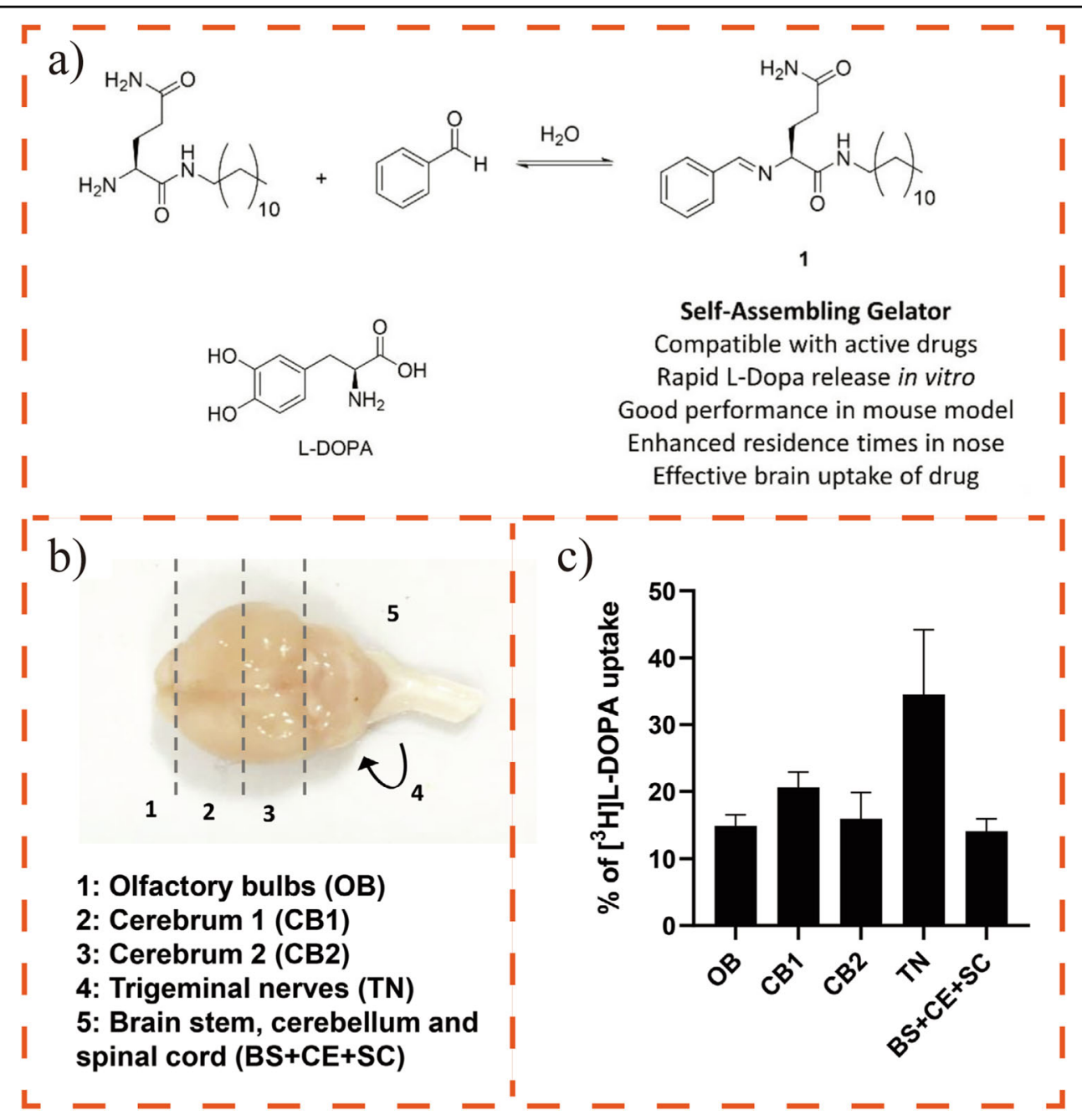

Fig. 5 Self-assembling hydrogels delivering L-dopa for the treatment of PD. a Schematic of the synthetic process of gelator with glutamine amide derivative and benzaldehyde, molecular structure of L-DOPA and superiorities of the gelator for nasal delivery. $\mathbf{b}$ Dorsal view of the brain and dissection locations of various brain parts including the olfactory bulbs (OB), the cerebrum (CB 1and 2), trigeminal nerves (TN), the brain stem (BS) and cerebellum (CE). c \% of [3H] L-DOPA taken by different brain segments. Whole body was perfused with $0.9 \%$ saline, and the studied tissues were dissected for liquid scintillation counting. Experimental results are expressed as \% uptake normalized to total [3H] L-DOPA. Data are expressed as the mean \pm SD, $n=3$. Reproduced with permission from ref. ${ }^{60}$. Copyright 2021 John Wiley and Sons.

caused by undesirable biochemical and/or immunological stress in vivo and changes in the environment from a $2 \mathrm{D}$ in vitro environment to a $3 \mathrm{D}$ in vivo environment after cell transplantation. To address these challenges, Schaffer's team transplanted human pluripotent stem cell (hPSC)-derived neurons loaded into optimized HA-based hydrogels functionalized by peptide chain GRGDNP (RGD) and heparin to target brain inflammation ${ }^{67}$. Compared with unencapsulated neurons, hydrogelencapsulated neurons exhibited approximately fivefold survival after implantation. In the second year, the team incorporated key biochemical cues such as neurotrophic factors or mitogenic factors into the hydrogel biomaterial and experimentally identified these factors after incorporating them into HA-based hydrogels for the construction of a dispersion-generating implantation platform (Fig. 6a, b) ${ }^{69}$. The introduced bioactive factors and well-designed hydrogels promoted the survival and dispersion of hPSC-derived neurons (Fig. 6c, d), mediating increased neurite outgrowth and graft innervation (Fig. 6g-i), alleviating the pathological symptoms in PD mice (Fig. 6e, f) and thus promoting the clinical translation of CRT for PD.

\section{Multifunctional hydrogels for brain tumor therapy}

Glioblastoma (GBM) is the most malignant tumor in the CNS and accounts for $16 \%$ of all tumors in the brain, leading to high mortality ${ }^{70,71}$. Most patients will experience disease progression within 12 months and die between 14 and 20 months after diagnosis ${ }^{72}$. 


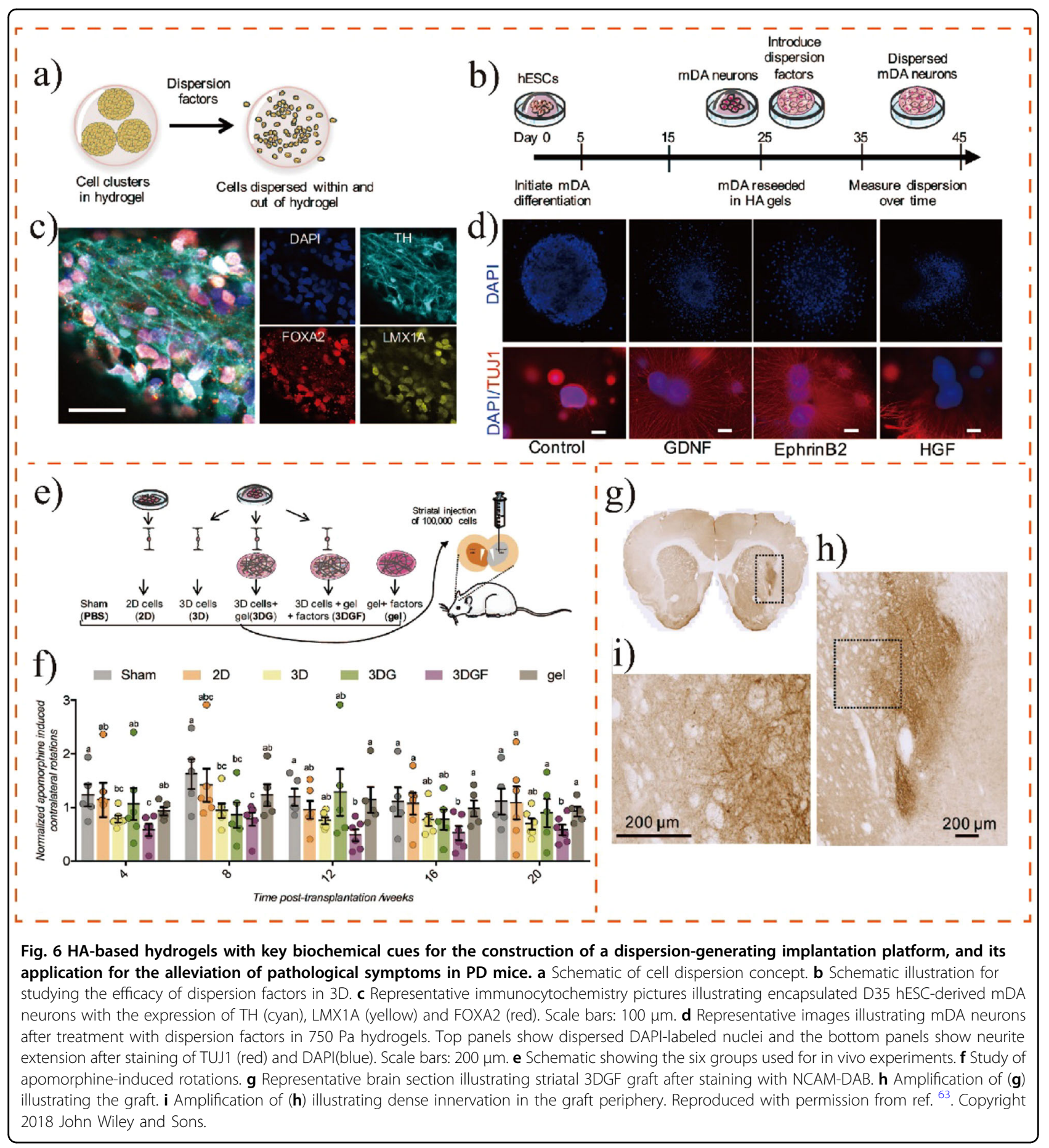

Current treatment methods for GBM include surgical resection followed by radiotherapy with temozolomide (TMZ) or TMZ-based chemotherapy. Unfortunately, these therapies have a modest effect on the inhibition of tumor recurrence and are at risk for eliciting systemic toxicity and off-target effects ${ }^{73}$. Given the cellular heterogeneity of GBM, alternative therapeutic methods are needed to strengthen clinical outcomes. Thus, the past decades are notable for the rapid development of hydrogels in GBM therapy because of some advantages of hydrogels, such as their noninvasiveness and sustained release of therapeutic agents after administration ${ }^{12}$. More importantly, these multifunctional hydrogels can be constructed with therapeutic agents and used as carriers for the drugs or as research models of the tumor microenvironment. 


\section{Hydrogels constructed with therapeutic agents}

Owing to the highly infiltrative properties, GBM margins are usually unclear, precluding complete resection of the GBM and causing recurrence in the vicinity of the surgical resection ${ }^{74,75}$. Early studies have found that tris(2-carboxyethyl) phosphine (TCEP) could regulate the oxidoreduction of cell surfaces to realize thiol-mediated cell adhesion, in which the disulfide bonds of cell surfaces could be reduced into free thiols to strengthen the adhesion between GBM and the $\mathrm{ECM}^{76}$. Based on these findings, Cha and Kim proposed a cancer cell-sticky hydrogel $(\mathrm{CSH})$ by immobilizing TCEP to modify the cell membrane of $\mathrm{GBM}^{77}$. The cancer cells could adhere to the CSH given the abundance of thiols at the cell surface, causing limited mobility and increased adhesion. In vivo xenograft models revealed that the fabricated hydrogels could decrease the invasiveness of GBM by trapping cancer cells. Chemical drugs can also inhibit the migration of GBM cells after surgical resection, but they have modest efficacy with exclusive use ${ }^{78}$. Bastianich et al. developed lauroyl-gemcitabine lipid-based $\left(\mathrm{GemC}_{12}-\mathrm{LNC}\right)$ hydrogels that could be administered in the resection cavity to bypass the $\mathrm{BBB}$, realizing high concentrations of the chemical drugs in the lesion site ${ }^{71}$. Research results suggested that after perisurgical injection in the resection cavity, $\mathrm{GemC}_{12}$ - $\mathrm{LNC}$ hydrogels effectively inhibited tumor recurrence and increased mouse survival. Brain tumor-initiating cells (BTICs) are one of the infiltrative cells that play a vital role in recurrence after tumor resection. Although camptothecin (CPT)-based chemotherapy eradicates residual BTICs, CPT has several limitations, including a short half-life and uneven tissue contact. To solve these problems, Schiapparelli et al. developed CPT prodrug-based hydrogels by the self-assembly method that can be used after tumor resection for local treatment (Fig. $7 \mathrm{a}, \mathrm{b})^{79}$. In vivo studies revealed that the CPT prodrug could be released into the brain parenchyma steadily in a resection mouse model, inhibiting tumor recurrence and prolonging the survival rate of mice (Fig. 7c). Peptide amphiphiles have provoked great interest in peptide-based drug composites because of their desirable self-assembling abilities. The delicate design has been applied to several therapeutic drugs, including CPT, doxorubicin (DOX), and PTX. Cui et al. have been working on drug-peptide conjugates over the past decade. They found that these amphiphiles exhibited excellent properties and wide applications in disease treatment and allowed the encapsulation of therapeutic agents for synergistic effects ${ }^{80,81}$. In 2019, Chakroun et al. fabricated PTX-peptide amphiphiles that could be spontaneously assembled into injectable supramolecular hydrogels ${ }^{82}$. Importantly, system disruption and molecular dissolution could regulate drug release. In addition, through molecular engineering of the hydrogels, such as changing the incorporation of hydrophobic segments, the drug release rate could be modified accurately. In cell studies, these PTX-peptide hydrogels exhibited tumor penetration in the tumor spheroid model, thus showing enhanced cytotoxicity against GBM.

In 2020, the team reported two CPT drug units linked with RGD for tumor tissue penetration that could associate with supramolecular polymers and form supramolecular tubustecan (TT) hydrogels following injection into tissues ${ }^{83}$. The hollowness of tubular assemblies allows the loading of DOX or curcumin for synergistic chemical therapy. In vivo studies have suggested that TT hydrogels with drug encapsulation promote tumor tissue penetration, allowing combination therapy to inhibit tumor metastasis and recurrence with reduced off-target side effects.

\section{Hydrogels as carriers for therapeutic agents}

Previous carriers of therapeutic drugs for GBM treatment may have had a high stiffness matrix, which is not good for prolonged release ${ }^{84}$. Higher stiffness will elicit side effects such as the sudden release of inclusions and the maladaptation of injected tissues ${ }^{85}$. Therefore, delivery devices with release timescales are needed for efficient clinical translation. Hydrogels are clinically effective vehicles for local delivery, in which HA-based hydrogels show notable potential in drug delivery because of their built-in biocompatibility and flexibility in composition and structure $^{86}$. Parkins et al. developed peptide-functionalized HAbased hydrogels as drug delivery reservoirs ${ }^{87}$. The assessment of the mechanical matching properties revealed a favorable adjustment of the designed hydrogels to local tissue stiffness, increasing the survival rate in human GBM models. Liposome-templated hydrogels (LHNs) have become an efficient vehicle for drug delivery. For example, Chen et al. designed an LHN nanoparticle for the codelivery of the clustered regularly interspaced short palindromic repeat (CRISPR)/Cas9 protein and nucleic acids ${ }^{88}$. Using polo-like kinase 1 as a model gene, in vivo studies have shown that LHNs are capable of delivering CRISPR/ Cas9 for tumor rejection.

Designing novel combination therapies that involve more than one drug for synergistic treatment has received increasing attention. To further improve the postsurgical treatment of GBM, Zhao developed photopolymerizable hydrogels for the codelivery of PTX and TMZ ${ }^{89}$. Combination therapy delivery could be realized by the system, and PTX in the hydrogel had sustained release for 1 month in mouse brains, eradicating tumors more significantly than single-drug therapy in the cancer model. Given the chronological order of biochemical pathways ${ }^{90}$, the temporal resolution of drugs in synergistic treatment greatly affects efficacy. A recent report demonstrated that the administration of erlotinib (ERL) and DOX in a timestaggered manner successfully depleted A549 lung cancer and BT-20 breast cancer cells, indicating that sequential 


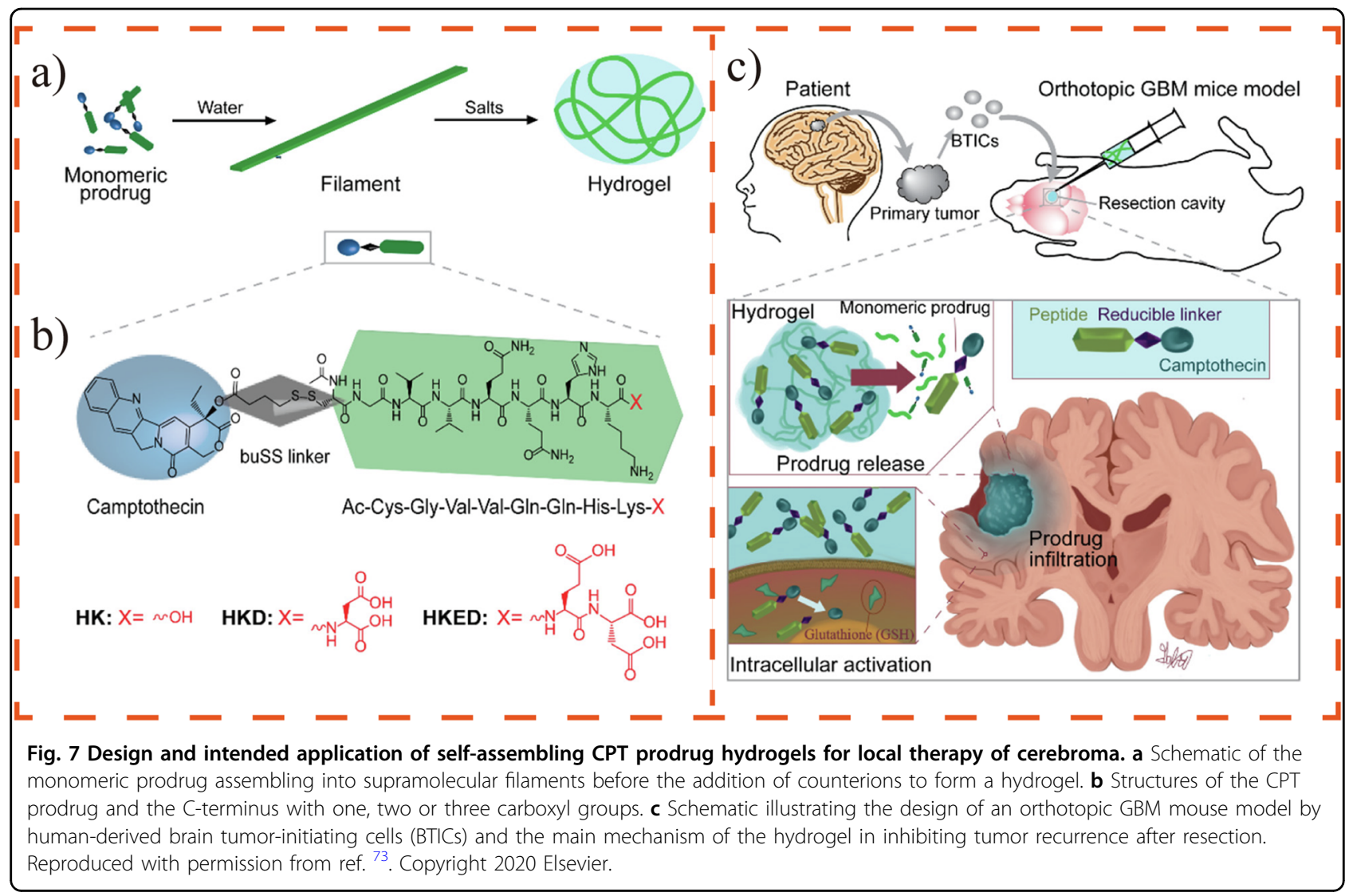

administration of DOX and ERL may be efficacious for GBM. Therefore, Majumder et al. fabricated multicompartment hydrogels composed of self-assembled peptides for the encapsulation of DOX and ERL and the delivery of these drugs in a time-resolved ERL-to-DOX manner ${ }^{91}$. The synergistic effect and sequential release of these two therapeutic agents exerted unparalleled efficacy in eliminating GBM.

\section{Hydrogel-based tissue engineering approaches}

Tissue damage and defects can lead to dysfunction. Since scientists first put forward the concept of "tissue engineering" in the 1980s, the possibility of its use in the treatment of patients with tissue defects or organ failure has been considered. Nevertheless, tissue engineering approaches include not only tissue regeneration but also tissue replacement and tissue-microenvironment simulation. For instance, tissue engineering approaches are typically used in the treatment of bone defects by promoting bone regeneration ${ }^{92}$. However, there are no effective therapies for the regeneration of injured cardiac tissue. Therefore, substitutes for engineered heart tissues were developed using tissue engineering approaches ${ }^{93}$. For GBM, tissue engineering approaches may be beneficial in simulating the tumor microenvironment, that is, the association between tumor tissue and the ECM.
Although some therapeutic agents, such as ERL, have a therapeutic effect on GBM, the efficacy is not durable for GBM with epidermal growth factor receptor (EGFR) mutations ${ }^{94}$. The intractable difficulties lie in the limited understanding of the relationship between the ECM and biophysical signals of the GBM with acquired resistance ${ }^{95}$. At present, signals from the ECM are involved in tumoracquired resistance, motivating researchers to replicate the complex tumor microenvironment to examine the processes related to EGFR resistance using tissue engineering approaches ${ }^{96}$. For example, to examine how combined signals of the ECM respond to ERL exposure, Pedron et al. used 3D gelatin hydrogels to simulate the GBM ECM, EGFR mutation status, and signals in response to ERL exposure ${ }^{97}$.

As GBM is highly vascularized, the internal influence of endothelial and cancer cells is actively involved in stimulating tumor growth ${ }^{98}$. Transwell assays are a common strategy for investigating tumor-endothelium interactions, but they insufficiently reflect the complex biochemical and physical microanatomical architecture in the tumor niche ${ }^{99}$. Recently, Wang et al. developed poly (ethylene glycol)-based hydrogels that can stimulate patient-derived GBM cells and mouse brain endothelial cells in a coculture model ${ }^{100}$. Alginate fibers were applied as porogens for the formation of vessel-like structures of 


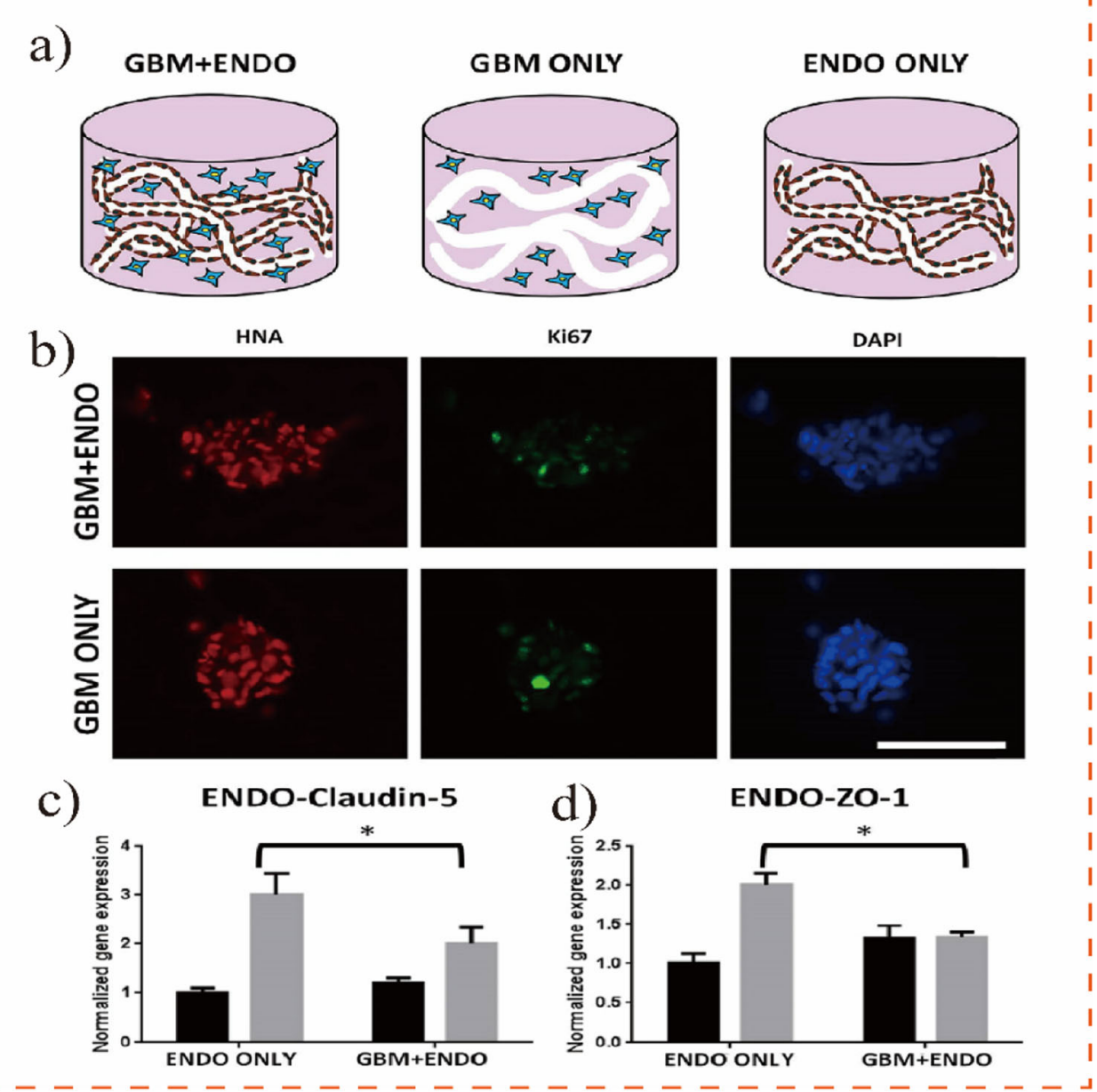

Fig. 8 Poly(ethylene glycol)-based hydrogels that can stimulate patient-derived GBM cells and mouse brain endothelial cells in a coculture model. a Schematic showing the experimental group design. Using degradable alginate microfibersas porogens, endothelial cells of mouse brain are made into vessel-like structures in a 3D biomimetic hydrogel. GBM patient-derived glioblastoma, ENDO endothelial cells. b Immunostaining with Ki67+ for proliferating tumor cells on Day 7. Human nuclear antigen (HNA), Ki67, and DAPI are stained to red, green and blue, respectively. Scale bar: $100 \mu \mathrm{m}$. c, d Expression of cell adhesion proteins in endothelial cells. Mouse specific primers are applied for the measurement of gene expression levels of claudin-5 and ZO-1 using RT-PCR. Reproduced with permission from ref. ${ }^{92}$. Copyright 2019 Elsevier.

endothelial cells in 3D hydrogels (Fig. 8a). Using the hydrogel system that could mimic the spatial organization of GBM and endothelial cells, researchers found that the growth of GBM cells was considerably increased (Fig. 8b), while the expression of cell adhesion proteins in endothelial cells was decreased (Fig. 8c, d) after the coculture. Therefore, the hydrogel system may help in the future design and development of tumor microenvironment simulations that are physiologically more similar to the actual microenvironment.

\section{Conclusions and perspectives}

CNS damage has long been an intractable challenge to tissue engineers because of the special physiological structure and complex pathophysiology that arise after injury ${ }^{101}$. Clinical strategies, including intrathecal drug delivery and stem cell transplantation, usually elicit the risk of local inflammatory responses and tissue damage. At present, CNS regenerative biomaterials focus on the local delivery of anti-ROS drugs and growth factors or the implantation of NSCs in a supporting scaffold. Therefore, the use of hydrogels is becoming unparalleled for drug delivery, as they realize high drug concentrations at the lesion site of the brain or spinal cord even with a low administration dose and minimal invasiveness. Hydrogels have various advantages that make them desirable materials for disease treatment, drug delivery, and even stem cell transplantation to the CNS.

This review summarizes how the special performance of hydrogels has achieved positive outcomes in many CNS disorders, such as brain injury, nerve damage, NDs, and brain tumors. For example, thermoresponsive 
hydrogels can be used as carriers of therapeutic drugs and functional cells for the treatment of NDs. Moreover, hydrogels can be constructed with therapeutic agents and used as carriers for therapeutic drugs, leading to localized drug release without systemic toxicity. Despite the satisfactory results of hydrogels for CNS delivery, better treatment outcomes should be pursued for the treatment of CNS diseases.

(1) Bionic concepts should be incorporated into the design of hydrogels. For example, some sophisticated chemical conjugations could be adopted to integrate biological ligands in the hydrogel scaffold. In this way, the elaborately designed hydrogel system will integrate well with the surrounding tissue, promoting cell migration, adhesion, and proliferation and thus exerting a better effect by promoting nerve injury recovery.

(2) Some of the latest technologies, such as artificial intelligence, should be used in the future design of smart hydrogels. In addition, bioinformatics and computational biology can help in the development of hydrogels because these tools can adjust the hydrophilicity of hydrogels, the hierarchical organizations of molecules, and the responses of hydrogels under various biological and mechanical stimuli.

(3) In the future, the release of the inclusions should be optimized by integrating hydrogels with other types of carriers, including liposomes and ionic polymers. This means that hierarchical drug delivery systems can be constructed to inhibit the rapid release in the initial hours after surgery, optimizing the pharmacodynamics of therapeutic agents.

In conclusion, hydrogels are potential scaffolds for both the treatment and the investigation of the mechanisms of CNS disorders. We anticipate the widespread use of this technology for the revolution of regenerative medicine and drug delivery systems.

\section{Acknowledgements}

We greatly acknowledge the financial support from the National Natural Science Foundation of China (No. 81901882), the China Postdoctoral Science Foundation (2019M663062), and Education Department of Henan Province (20A430026).

\section{Author contributions}

Q.L. and W.J.: paper preparation, figure and table preparation, and paper editing and revision. Y.L. and X.S.: literature collection and evaluation and paper draft preparation. X.D., Q.G., and B.Y.: figure legend preparation, reference insertion, and graphic abstract preparation.

\section{Competing interests}

The authors declare no competing interests.

\section{Publisher's note}

Springer Nature remains neutral with regard to jurisdictional claims in published maps and institutional affiliations.
Received: 28 August 2021 Revised: 10 January 2022 Accepted: 24 January 2022.

Published online: 25 February 2022

\section{References}

1. Abbott, N. J., Pizzo, M. E., Preston, J. E., Janigro, D. \& Thorne, R. G. The role of brain barriers in fluid movement in the CNS: is there a 'glymphatic' system? Acta Neuropathol. 135, 387-407 (2018).

2. Sweeney, M. D., Sagare, A. P. \& Zlokovic, B. V. Blood-brain barrier breakdown in Alzheimer disease and other neurodegenerative disorders. Nat. Rev. Neurol. 14, 133-150 (2018).

3. Dong, X. Current strategies for brain drug delivery. Theranostics 8, 1481-1493 (2018).

4. Tosi, G., Costantino, L., Ruozi, B., Forni, F. \& Vandelli, M. A. Polymeric nanoparticles for the drug delivery to the central nervous system. Expert Opin. Drug Deliv. 5, 155-174 (2008).

5. Jacobs, W. B. \& Fehlings, M. G. The molecular basis of neural regeneration. Neurosurgery 53, 943-948 (2003). discussion 948-950.

6. Ran, W. \& Xue, X. Theranostical application of nanomedicine for treating central nervous system disorders. Sci. China Life Sci. 61, 392-399 (2018)

7. Lisik K. \& Krokosz A. Application of carbon nanoparticles in oncology and regenerative medicine. Int. J. Mol. Sci. 22, 8341 (2021).

8. Guan, Y. et al. Ceria/POMs hybrid nanoparticles as a mimicking metallopeptidase for treatment of neurotoxicity of amyloid-beta peptide. Biomaterials 98, 92-102 (2016).

9. Mason, C. \& Dunnill, P. A brief definition of regenerative medicine. Regen. Med. 3, 1-5 (2008).

10. $\mathrm{Yu}, \mathrm{D}$. et al. MOF-encapsulated nanozyme enhanced siRNA combo: control neural stem cell differentiation and ameliorate cognitive impairments in Alzheimer's disease model. Biomaterials 255, 120160 (2020).

11. Sun, $\mathrm{H}$. et al. Wireless near-infrared electrical stimulation of neurite outgrowth. Chem. Commun. 55, 9833-9836 (2019).

12. Bellotti, E., Schilling, A. L., Little, S. R. \& Decuzzi, P. Injectable thermoresponsive hydrogels as drug delivery system for the treatment of central nervous system disorders: a review. J. Control Release 329, 16-35 (2021).

13. Pakulska, M. M., Ballios, B. G. \& Shoichet, M. S. Injectable hydrogels for central nervous system therapy. Biomed. Mater. 7, 024101 (2012).

14. Barchet, T. M. \& Amiji, M. M. Challenges and opportunities in CNS delivery of therapeutics for neurodegenerative diseases. Expert Opin. Drug Deliv. 6, 211-225 (2009).

15. Oller-Salvia, B., Sanchez-Navarro, M., Giralt, E. \& Teixido, M. Blood-brain barrier shuttle peptides: an emerging paradigm for brain delivery. Chem. Soc. Rev. 45, 4690-4707 (2016).

16. Kou, L. et al. L-Carnitine-conjugated nanoparticles to promote permeation across blood-brain barrier and to target glioma cells for drug delivery via the novel organic cation/carnitine transporter OCTN2. Artif. Cells Nanomed. Biotechnol. 46, 1605-1616 (2018).

17. Akhtar, A. et al. Neurodegenerative diseases and effective drug delivery: A review of challenges and novel therapeutics. J. Control Release 330, 1152-1167 (2021).

18. Uchida, Y., Zhang, Z., Tachikawa, M. \& Terasaki, T. Quantitative targeted absolute proteomics of rat blood-cerebrospinal fluid barrier transporters: comparison with a human specimen. J. Neurochem. 134, 1104-1115 (2015).

19. Lu, C. T. et al. Current approaches to enhance CNS delivery of drugs across the brain barriers. Int. J. Nanomed. 9, 2241-2257 (2014).

20. Stockwell, J., Abdi, N., Lu, X., Maheshwari, O. \& Taghibiglou, C. Novel central nervous system drug delivery systems. Chem. Biol. Drug Des. 83, 507-520 (2014).

21. Kasinathan, N., Jagani, H. V., Alex, A. T., Volety, S. M. \& Rao, J. V. Strategies for drug delivery to the central nervous system by systemic route. Drug Deliv. 22, 243-257 (2015).

22. Kumari, A., Yadav, S. K. \& Yadav, S. C. Biodegradable polymeric nanoparticles based drug delivery systems. Colloids Surf. B Biointerfaces 75, 1-18 (2010).

23. De, WitteE. et al. A valid alternative for in-person language assessments in brain tumor patients: feasibility and validity measures of the new TeleLanguage test. Neurooncol Pract. 6, 93-102 (2019).

24. Rogawski, M. A. Convection-enhanced delivery in the treatment of epilepsy. Neurotherapeutics 6, 344-351 (2009). 
25. Yin, D. et al. Convection-enhanced delivery improves distribution and efficacy of tumor-selective retroviral replicating vectors in a rodent brain tumor model. Cancer Gene Ther. 20, 336-341 (2013)

26. Qu, Y. et al. Injectable and thermosensitive hydrogel and PDLLA electrospun nanofiber membrane composites for guided spinal fusion. ACS Appl. Mater. Interfaces 10, 4462-4470 (2018).

27. Lee J. \& Kang S. K. Principles for Controlling the Shape Recovery and Degradation Behavior of Biodegradable Shape-Memory Polymers in Biomedical Applications. Micromachines 12, 757 (2021)

28. Weber, L. M., Lopez, C. G. \& Anseth, K. S. Effects of PEG hydrogel crosslinking density on protein diffusion and encapsulated islet survival and function. J. Biomed. Mater. Res A. 90, 720-729 (2009).

29. Baumann, M. D., Kang, C. E., Tator, C. H. \& Shoichet, M. S. Intrathecal delivery of a polymeric nanocomposite hydrogel after spinal cord injury. Biomaterials 31, 7631-7639 (2010)

30. Van Tomme, S. R., Storm, G. \& Hennink, W. E. In situ gelling hydrogels for pharmaceutical and biomedical applications. Int J. Pharm. 355, 1-18 (2008).

31. Ulndreaj, A., Badner, A.\& Fehlings, M. G. Promising neuroprotective strategies for traumatic spinal cord injury with a focus on the differential effects among anatomical levels of injury. F1000Res. 6, 1907 (2017)

32. Kim, S. et al. The inhibition of glioma growth in vitro and in vivo by a chitosan/ellagic acid composite biomaterial. Biomaterials 30, 4743-4751 (2009)

33. Zou J. L. Peripheral nerve-derived matrix hydrogel promotes remyelination and inhibits synapse formation. Adv. Funct. Mater. 28, 1705739 (2018).

34. Hyder, A. A., Wunderlich, C. A., Puvanachandra, P., Gururaj, G. \& Kobusingye, O. C. The impact of traumatic brain injuries: a global perspective. NeuroRehabilitation 22, 341-353 (2007).

35. Fernandez-Gajardo, R. et al. Novel therapeutic strategies for traumatic brain injury: acute antioxidant reinforcement. CNS Drugs 28, 229-248 (2014).

36. Qian, F. et al. In Situ implantable, post-trauma microenvironment-responsive, ROS Depletion Hydrogels for the treatment of Traumatic brain injury. Biomaterials 270, 120675 (2021).

37. Duncan, T. \& Valenzuela, M. Alzheimer's disease, dementia, and stem cel therapy. Stem Cell Res. Ther. 8, 111 (2017).

38. Zhang, K. Potential application of an injectable hydrogel scaffold loaded with mesenchymal stem cells for treating traumatic brain injury. J. Mater. Chem. B. 6. 2982-2992 (2018).

39. Sultan, M. T. Reinforced-hydrogel encapsulated hMSCs towards brain injury treatment by trans-septal approach. Biomaterials 266, 120413 (2021).

40. Silver F. H., Kelkar N. \& Deshmukh T. Molecular basis for mechanical properties of ECMs: proposed role of fibrillar collagen and proteoglycans in tissue biomechanics. Biomolecules 11, 1018 (2021).

41. Wang, T. W. et al. Effects of an injectable functionalized self-assembling nanopeptide hydrogel on angiogenesis and neurogenesis for regeneration of the central nervous system. Nanoscale 9, 16281-16292 (2017).

42. Silva, N. A., Sousa, N., Reis, R. L. \& Salgado, A. J. From basics to clinical: a comprehensive review on spinal cord injury. Prog. Neurobiol. 114, 25-57 (2014).

43. Shultz, R. B. \& Zhong, Y. Minocycline targets multiple secondary injury mechanisms in traumatic spinal cord injury. Neural Regen. Res. 12, 702-713 (2017).

44. Lanska, D. J. The influence of the two world wars on the development of rehabilitation for spinal cord injuries in the United States and Great Britain. Front. Neurol. Neurosci. 38, 56-67 (2016).

45. Assuncao-Silva, R. C., Gomes, E. D., Sousa, N., Silva, N. A. \& Salgado, A. J. Hydrogels and cell based therapies in spinal cord injury regeneration. Stem Cells Int. 2015, 948040 (2015).

46. Nazemi, Z. et al. Co-delivery of minocycline and paclitaxel from injectable hydrogel for treatment of spinal cord injury. J. Control Release 321, 145-158 (2020)

47. Sabelstrom, H., Stenudd, M. \& Frisen, J. Neural stem cells in the adult spinal cord. Exp. Neurol. 260, 44-49 (2014).

48. Yang, Y. et al. Small molecules combined with collagen hydrogel direct neurogenesis and migration of neural stem cells after spinal cord injury Biomaterials 269, 120479 (2021).

49. Chen, W. C. et al. Transplantation of mesenchymal stem cells for spinal cord injury: a systematic review and network meta-analysis. J. Transl. Med. 19, 178 (2021).
50. Yuan T. Highly permeable DNA supramolecular hydrogel promotes neurogenesis and functional recovery after completely transected spinal cord injury. Adv Mater. https://doi.org/10.1002/adma.202102428e2102428 (2021).

51. Aly, A. E. et al. Intranasal delivery of pGDNF DNA nanoparticles provides neuroprotection in the rat 6-hydroxydopamine model of Parkinson's disease. Mol. Neurobiol. 56, 688-701 (2019).

52. Sabir F. Development and characterization of n-propyl gallate encapsulated solid lipid nanoparticles-loaded hydrogel for intranasal delivery. Pharmaceuticals 14, 696 (2021).

53. Li, Y., Li, J., Zhang, X., Ding, J. \& Mao, S. Non-ionic surfactants as novel intranasal absorption enhancers: in vitro and in vivo characterization. Drug Deliv. 23, 2272-2279 (2016)

54. Pathak, R., Dash, R. P., Misra, M. \& Nivsarkar, M. Role of mucoadhesive polymers in enhancing delivery of nimodipine microemulsion to brain via intranasal route. Acta Pharm. Sin. B. 4, 151-160 (2014).

55. Grover, S., Sahoo, S., Chakrabarti, S. \& Avasthi, A. Post-traumatic stress disorder (PTSD) related symptoms following an experience of delirium. J. Psychosom. Res. 123, 109725 (2019).

56. Pang, L. et al. Intranasal temperature-sensitive hydrogels of cannabidio inclusion complex for the treatment of post-traumatic stress disorder. Acta Pharmaceutica Sin. B. 11, 2031-2047 (2021).

57. Wang, Q. S. et al. Intranasal delivery of berberine via in situ thermoresponsive hydrogels with non-invasive therapy exhibits better antidepressant-like effects. Biomater. Sci. 8, 2853-2865 (2020).

58. Jiang, Y. Intranasal brain-derived neurotrophic factor protects brain from ischemic insult via modulating local inflammation in rats. Neuroscience 172, 398-405 (2011).

59. Su, X., Huang, L., Xiao, D., Qu, Y. \& Mu, D. Research progress on the role and mechanism of action of activin A in brain injury. Front Neurosci. 12, 697 (2018)

60. Steidinger, T. U., Slone, S. R., Ding, H., Standaert, D. G. \& Yacoubian, T. A. Angiogenin in Parkinson disease models: role of Akt phosphorylation and evaluation of AAV-mediated angiogenin expression in MPTP treated mice. PLOS ONE 8, e56092 (2013).

61. Sun, $Y$. et al. Functional self-assembling peptide nanofiber hydrogels designed for nerve degeneration. ACS Appl Mater. Interfaces 8, 2348-2359 (2016)

62. Guo, J. et al. Self-assembling peptide nanofiber scaffold promotes the reconstruction of acutely injured brain. Nanomedicine 5, 345-351 (2009).

63. Adak, A. Biodegradable neuro-compatible peptide hydrogel promotes neurite outgrowth, shows significant neuroprotection, and delivers antiAlzheimer drug. ACS Appl Mater. Interfaces 9, 5067-5076 (2017).

64. Clarkin, O. M. et al. Novel injectable gallium-based self-setting glass-alginate hydrogel composite for cardiovascular tissue engineering. Carbohydr. Polym. 217, 152-159 (2019)

65. Li, J. et al. A drug delivery hydrogel system based on activin B for Parkinson's disease. Biomaterials 102, 72-86 (2016).

66. Wang, J. T. et al. Enhanced delivery of neuroactive drugs via nasal delivery with a self-healing supramolecular gel. Adv. Sci. 8, e2101058 (2021).

67. Adil, M. M. et al. Engineered hydrogels increase the post-transplantation survival of encapsulated hESC-derived midbrain dopaminergic neurons. Biomaterials 136, 1-11 (2017)

68. Moriarty, N., Cabre, S., Alamilla, V., Pandit, A. \& Dowd, E. Encapsulation of young donor age dopaminergic grafts in a GDNF-loaded collagen hydrogel further increases their survival, reinnervation, and functional efficacy after intrastriatal transplantation in hemi-Parkinsonian rats. Eur. J. Neurosci. 49 487-496 (2019).

69. Adil M. M. Dopaminergic neurons transplanted using cell-instructive biomaterials alleviate parkinsonism in rodents. Adv. Funct. Mater. 28, 1804144 (2018)

70. Wei, X., Chen, X., Ying, M. \& Lu, W. Brain tumor-targeted drug delivery strategies. Acta Pharm. Sin. B. 4, 193-201 (2014).

71. Bastiancich, $C$. et al. Injectable nanomedicine hydrogel for local chemotherapy of glioblastoma after surgical resection. J. Control Release $\mathbf{2 6 4}$ 45-54 (2017).

72. Medikonda $\mathrm{R}$, et al. Synergy between glutamate modulation and antiprogrammed cell death protein 1 immunotherapy for glioblastoma. J. Neurosurg. https://doi.org/10.3171/2021.1.JNS2024821-10 (2021).

73. Tsao, C. T. Thermoreversible poly(ethylene glycol)-g-chitosan hydrogel as a therapeutic T lymphocyte depot for localized glioblastoma immunotherapy. Biomacromolecules 15, 2656-2662 (2014). 
74. Huse, J. T. \& Holland, E. C. Targeting brain cancer: advances in the molecular pathology of malignant glioma and medulloblastoma. Nat. Rev. Cancer 10 319-331 (2010).

75. Bellail, A. C., Hunter, S. B., Brat, D. J., Tan, C. \& Van Meir, E. G. Microregional extracellular matrix heterogeneity in brain modulates glioma cell invasion. Int J. Biochem. Cell Biol. 36, 1046-1069 (2004).

76. Moon $\mathrm{H}$. et al. Delta(8(14))-ergostenol glycoside derivatives inhibit the expression of inflammatory mediators and matrix metalloproteinase. Molecules 26, 4547 (2021).

77. Cha, J. \& Kim, P. Cancer cell-sticky hydrogels to target the cell membrane of invading glioblastomas. ACS Appl Mater. Interfaces 13, 31371-31378 (2021).

78. Osuka, S. \& Van Meir, E. G. Overcoming therapeutic resistance in glioblastoma: the way forward. J. Clin. Investig. 127, 415-426 (2017)

79. Schiapparelli, P. et al. Self-assembling and self-formulating prodrug hydrogelator extends survival in a glioblastoma resection and recurrence model. J. Control Release 319, 311-321 (2020).

80. Cheetham, A. G., Zhang, P., Lin, Y. A., Lin, R. \& Cui, H. Synthesis and selfassembly of a Mikto-Arm star dual drug amphiphile containing both paclitaxel and camptothecin. J. Mater. Chem. B. 2, 7316-7326 (2014).

81. Lin, R., Cheetham, A. G., Zhang, P., Lin, Y. A. \& Cui, H. Supramolecular filaments containing a fixed $41 \%$ paclitaxel loading. Chem. Commun. 49, 4968-4970 (2013).

82. Chakroun, R. W. et al. Fine-tuning the linear release rate of paclitaxel-bearing supramolecular filament hydrogels through molecular engineering. ACS Nano. 13, 7780-7790 (2019).

83. Wang, F. et al. Supramolecular tubustecan hydrogel as chemotherapeutic carrier to improve tumor penetration and local treatment efficacy. ACS Nano. 14, 10083-10094 (2020).

84. Ashby, L. S., Smith, K. A. \& Stea, B. Gliadel wafer implantation combined with standard radiotherapy and concurrent followed by adjuvant temozolomide for treatment of newly diagnosed high-grade glioma: a systematic literature review. World J. Surg. Oncol. 14, 225 (2016).

85. Sage, W. et al. Local alkylating chemotherapy applied immediately after 5-ALA guided resection of glioblastoma does not provide additional benefit. J. Neurooncol. 136, 273-280 (2018).

86. Rowland, M. J., Atgie, M., Hoogland, D. \& Scherman, O. A. Preparation and supramolecular recognition of multivalent peptide-polysaccharide conjugates by cucurbit[8]uril in hydrogel formation. Biomacromolecules $\mathbf{1 6}$ 2436-2443 (2015).

87. Parkins C. C. et al. Mechanically matching the rheological properties of brain tissue for drug-delivery in human glioblastoma models. Biomaterials. https:// doi.org/10.1016/j.biomaterials.2021.120919 (2021).
88. Chen Z. et al. Targeted delivery of CRISPR/Cas9-mediated cancer gene therapy via liposome-templated hydrogel nanoparticles. Adv. Funct. Mater. 27, 1703036 (2017).

89. Zhao, M. Codelivery of paclitaxel and temozolomide through a photopolymerizable hydrogel prevents glioblastoma recurrence after surgica resection. J. Control Release 309, 72-81 (2019).

90. Aboseada, H. A., Hassanien, M. M., El-Sayed, I. H. \& Saad, E. A. Schiff base 4ethyl-1-(pyridin-2-yl) thiosemicarbazide up-regulates the antioxidant status and inhibits the progression of Ehrlich solid tumor in mice. Biochem Biophys. Res. Commun. 573, 42-47 (2021).

91. Majumder, P., Baxa, U., Walsh, S. T. R. \& Schneider, J. P. Design of a multicompartment hydrogel that facilitates time-resolved delivery of combination therapy and synergized killing of glioblastoma. Angew. Chem. Int. Ed. Engl. 57, 15040-15044 (2018).

92. Kim H. D. et al. Biomimetic materials and fabrication approaches for bone tissue engineering. Adv. Healthc. Mater. 6, 1700612 (2017).

93. Hendrickson, T. et al. Mimicking cardiac tissue complexity through physical cues: A review on cardiac tissue engineering approaches. Nanomedicine 33, 102367 (2021).

94. Padfield, E., Ellis, H. P. \& Kurian, K. M. Current therapeutic advances targeting EGFR and EGFRvIII in glioblastoma. Front. Oncol. 5, 5 (2015).

95. Jackman, D. et al. Clinical definition of acquired resistance to epidermal growth factor receptor tyrosine kinase inhibitors in non-small-cell lung cancer. J. Clin. Oncol. 28, 357-360 (2010).

96. Pedron S., Hanselman J. S., Schroeder M. A., Sarkaria J. N. \& Harley B. A. C. Extracellular hyaluronic acid influences the efficacy of EGFR tyrosine kinase inhibitors in a biomaterial model of glioblastoma. Adv. Healthc. Mater. 6 , 1700529 (2017).

97. Pedron, S. et al. Hyaluronic acid-functionalized gelatin hydrogels reveal extracellular matrix signals temper the efficacy of erlotinib against patient-derived glioblastoma specimens. Biomaterials 219, 119371 (2019).

98. Hardee, M. E. \& Zagzag, D. Mechanisms of glioma-associated neovascularization. Am. J. Pathol. 181, 1126-1141 (2012).

99. Vaupel, P. \& Harrison, L. Tumor hypoxia: causative factors, compensatory mechanisms, and cellular response. Oncologist 9(Suppl 5), 4-9 (2004).

100. Wang, C. et al. Mimicking brain tumor-vasculature microanatomical architecture via co-culture of brain tumor and endothelial cells in 3D hydrogels. Biomaterials 202, 35-44 (2019).

101. Mukherjee, N., Adak, A. \& Ghosh, S. Recent trends in the development of peptide and protein-based hydrogel therapeutics for the healing of CNS injury. Soft Matter 16, 10046-10064 (2020). 